\title{
Automated quantification with BRASS reduces equivocal reporting of DaTSCAN ('23-FP-CIT) SPECT studies
}

Deborah Ruth Pencharz, Paul Hanlon, Riddhika Chakravartty, Shaunak Navalkissoor, Ann-Marie Quigley, Margaret Hall, Thomas Wagner Royal Free London NHS Foundation Trust, United Kingdom

There are no conflicts of interest in this work.

[Received 9 XII 2013; Accepted 7 VII 2014]

\begin{abstract}
BACKGROUND: ${ }^{123}$-FP-CIT (DaTSCAN) SPECT studies of the nigrostriatal pathway are a valuable tool in the diagnosis of movement disorders. However some scans are reported as equivocal with potential adverse consequences. We investigated whether the use of quantification of tracer uptake within the striatum can be used to reduce the number of equivocal reports. MATERIAL AND METHODS: BRASS software (Hermes, Sweden) was used to quantify striatal tracer uptake in DaTSCAN studies of patients referred to our institution. Scans were quantified and numerical limits were determined to distinguish between normal and abnormal scans. Scans were then re-reported both with, and without, the use of quantification. Number of equivocal reports and accuracy of reporting between the two types of reporting were compared.

RESULTS: Scan reporting using quantification led to a significant reduction in the number of equivocal reports with no significant change in reporting accuracy.

CONCLUSION: Automated quantification of DaTSCAN studies with BRASS and the use of numerical limits can decrease the number of equivocal reports without affecting report accuracy.
\end{abstract}

KEY words: DaTSCAN, quantification, Parkinson's disease, diagnosis

Nuclear Med Rev 2014; 17, 2: 65-69

\section{Introduction}

The diagnosis of idiopathic Parkinson's disease (PD) and other Parkinson plus syndromes (PS) relies primarily on clinical assessment [1]. However in cases of diagnostic uncertainty, such as distinguishing PD from other causes of tremor or from Parkinsonian symptoms associated with neuroleptic exposure dopamine transporter, single photon emission computed tomography (SPECT) using ${ }^{123}$ I-FP-CIT (DaTSCAN) is useful and recommended $[1,2]$.

${ }^{123}$ I-FP-CIT (DaTSCAN) binds to pre-syxnaptic dopamine transporters in the striatum. If tracer uptake in either or both striata is reduced and increased background activity is seen, as shown in Figure 1, then the scan is reported as abnormal and there is a high probability that the patient has PD or a PS. If the uptake in the stria-

Correspondence to: Deborah Ruth Pencharz, MD

Royal Free London NHS Foundation Trust

Department of Nuclear Medicine

Royal Free Hospital

Pond Street, London, NW3, UK

Phone: (+44) 7545077713

E-mail: debpencharz@hotmail.com tum is normal, as shown in Figure 2, then this makes a diagnosis of PD or a PS unlikely [3]. In some scans, it is difficult to determine if tracer uptake is normal or reduced and these scans are usually reported as equivocal. Figure 3 shows a scan with reduced uptake on the left, but this is not obviously abnormal and may be reported as equivocal. Most scans are clearly normal or abnormal, however some are more difficult to interpret and approximately $8 \%$ to $9 \%$ of DaTSCANS are reported as equivocal [4 and data from our department]. This has the adverse consequences of delayed definitive diagnosis, possible delay in treatment and may also result in a repeat scan causing increased radiation exposure and anxiety to the patient and increased cost to the healthcare provider.

A number of software packages exist that allow quantification of uptake in the striata [5] and different methods of quantification exist and have been compared [6]. Quantification has been shown to reduce interobserver variability in scan reporting [7] and to correlate with visual assessment [8], however to our knowledge no studies have specifically studied whether quantification can reduce the number of scans reported as equivocal. The aim of our study was to determine numerical limits from the results of quantification that could be used to help define scans that appear equivocal as normal or abnormal. 


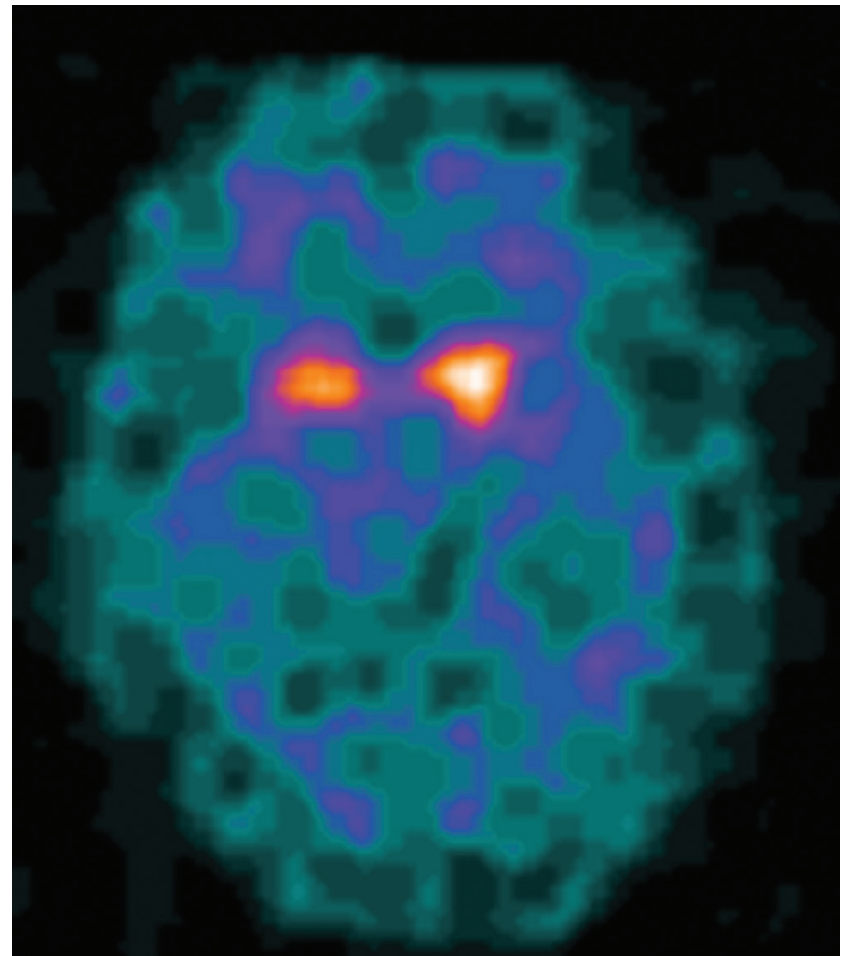

Figure 1. Abnormal DaTSCAN with reduced uptake in the striata, particularly the putamina, bilaterally and increased background activity

\section{Materials and methods}

\section{Study design and patient selection}

As post mortem diagnosis is rarely available and, according to recent recommendations, the diagnosis of $\mathrm{PD}$ is still predominantly based on the correct identification of its clinical features [1], we used clinical follow up as the gold standard for disease diagnosis. We chose a minimum of 18 months follow up between scan and final diagnosis, which was taken from the most recent correspondence letter in the clinical notes. Our department performed 611 scans between 2006 and 2009, of these 110 had follow up greater than 18 months. One scan was excluded from the study as correlative imaging showed that the patient had had a basal ganglia infarct which caused marked unilateral reduction in uptake, although the patient did not have a diagnosis consistent with PD or a PS. Mean follow-up period was 3.3 years and maximum was 6.7 years.

Each of the 109 scans was re-reported by three nuclear medicine consultants and two non-clinical members of the nu-

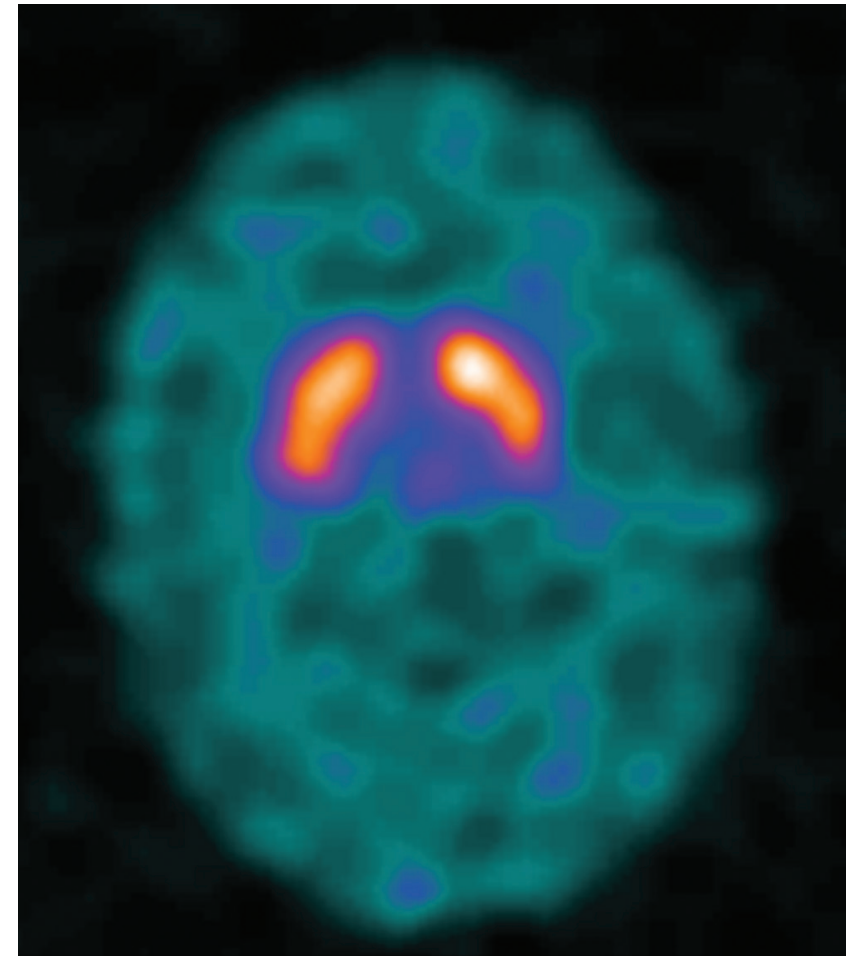

Figure 2. Normal DaTSCAN with good uptake in all of the striata bilaterally and normal background activity

clear medicine team (physicists) with little previous experience of DaTSCAN interpretation. The non-clinical assessors were given a short tutorial on DaTSCAN reporting prior to the exercise. The scans were re-reported in two rounds, firstly without the results of quantification of uptake in the striata and secondly with the results of quantification. Each round of reporting was blinded to the results of previous reports, clinical details and the patient diagnosis and the order in which the scans were reported was changed. The assessors were asked to report the scans as normal, abnormal or equivocal.

\section{Scan acquisition and processing}

Patients were given an intravenous injection of $\sim 185 \mathrm{MBq}$ of loflupane ([l-123] N- $\omega$-fluoropropyl-2 $\beta$-carbomethoxy-3 $\beta$-(4-iodophenyl) nortropane/DaTSCAN (GE Healthcare)). Images were taken 3-4 hours post-injection, using a Philips IRIX 3 headed camera, a $128 \times 128$ matrix, 30 seconds per step, 3 degrees per step, low energy high resolution collimator, $20 \%$ energy window and
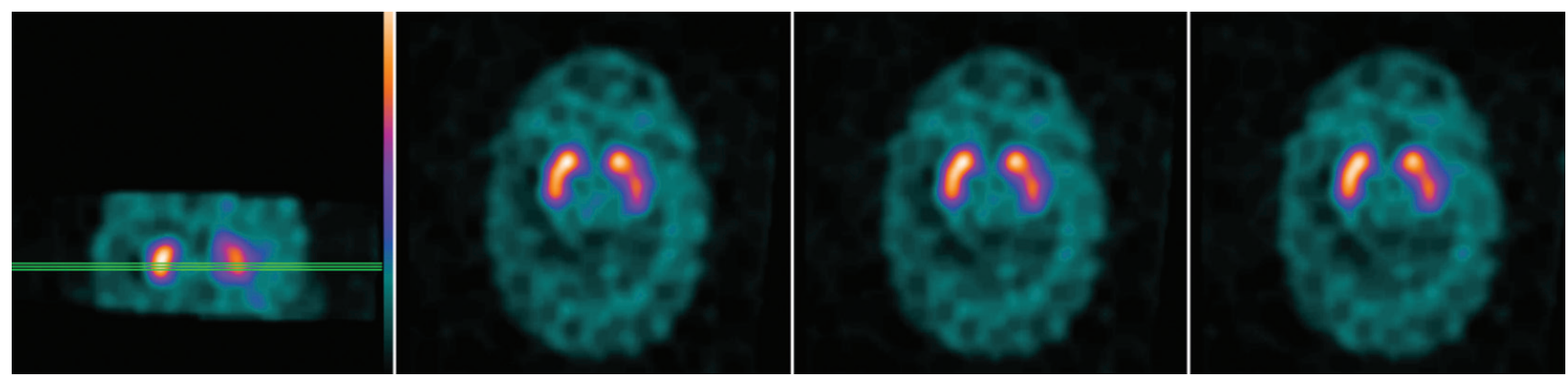

Figure 3. A scan that might be reported as equivocal 
magnification $\times 2$. Scans were processed on a Philips Odyssey, re-constructed iteratively (OS ML-EM-all) and post-filtered with a low pass Butterworth filter of order 4 and a cut-off frequency of 0.3 cycles/2 pixels.

\section{Scan quantification}

The Brain Registration \& Analysis Software Suite (BRASS"', HERMES Medical, Sweden) was used to quantify tracer uptake in the putamina and caudate nuclei [9]. This software has been shown to provide consistent results independent of operator variability [10]. BRASS fits the patient data to a pre-existing template containing a number of volumes of interest (VOIs) and calculates the caudate and putamen uptake ratios (CUR and PUR respectively),

$$
C U R=\frac{C-B}{B} \quad \text { and } \quad P U R=\frac{P-B}{B}
$$

where $C, P$ and $B$ are the average counts per voxel in the caudate nucleus, putamen and background regions, respectively. The background region is a reference volume within the occipital lobe.

The PUR and CUR in the striatum bilaterally in normal and abnormal scans were compared. Minimum CUR and PUR (CUR ${ }_{\text {min }}$, $\left.P U R_{\text {min }}\right)$ were determined above which all normal scans lay. If the lowest value for the CUR or PUR, on either side of a scan fell

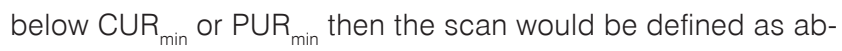
normal. The converse was not true: if the lowest PUR or CUR fell ${\text { above } \text { CUR }_{\text {min }} \text { or PUR }}_{\text {min }}$, the scan could be normal or abnormal. The number of scans correctly classified as abnormal with use of either $\mathrm{CUR}_{\text {min }}$ or $\mathrm{PUR}_{\text {min }}$ was compared and the parameter that detected the most number of abnormal scans was used in the reporting exercise.

\section{Comparison of reporting with and without quantification}

For each round of reporting the number of equivocal reports and accuracy of reporting was recorded and compared. Accuracy was calculated as the percentage of scans reported as normal or abnormal which agreed with clinical follow up (scans reported as equivocal were excluded from the calculation). Accuracy was calculated so that if the use of quantification resulted in less scans being reported as equivocal, then we could determine if the decision about whether the scan was normal or abnormal was actually correct. Because CUR ${ }_{\text {min }}$ and PUR ${ }_{\text {min }}$ were determined, and then used, on the same population of scans it was not appropriate to compare accuracy of reporting between the two rounds as an independent result.

\section{Results}

109 patients were included in the study. There were 59 men and 50 women; mean age was 68 years. $77 \%(n=84)$ of patients were diagnosed (on clinical follow up) with PD or a PS, 33\% ( $n=25)$ of patients were diagnosed with a range of other disorders including essential tremor, vascular and drug induced parkinsonism.

Figure 4 shows the uptake ratios in the putamen and caudate nucleus in normal and abnormal scans. The $\mathrm{CUR}_{\text {min }}$ or PUR $\mathrm{R}_{\text {min }}$ for which all normal scans were equal to or lay above was 1.43 and
1.41 respectively. Rather than use 1.42 and 1.40 as the cut off for which scans lying below these values are classified as abnormal, we used the values of 1.37 and 1.35 to give a margin of error.

The mean caudate uptake ratio in the group with PD or PS is approximately $61 \%$ of that measured in the non PD/PS group. For the putamina this value is $37 \%$. This greater reduction in the mean signal in the putamen is due to neuronal degeneration affecting this part of the striatum first [11].

Table 1 shows the number of abnormal scans identified by CUR $_{\text {min }}$ and PUR min $_{\text {min }}$ CUR $_{\text {min }}$ did not classify any scans as abnormal that had not already been classified as abnormal with the use of $\mathrm{PUR}_{\text {min }}$. Therefore PUR $\mathrm{Rin}_{\text {min }}$ only was used in the reporting exercise. Tables 2A and 2B show the results of the two rounds of reporting.

Mean number of equivocal reports without quantification was 10.6 (SD 2.3) and with quantification was 3.6 (SD 1.7). Student's t-test (two tailed, paired) showed that the number of
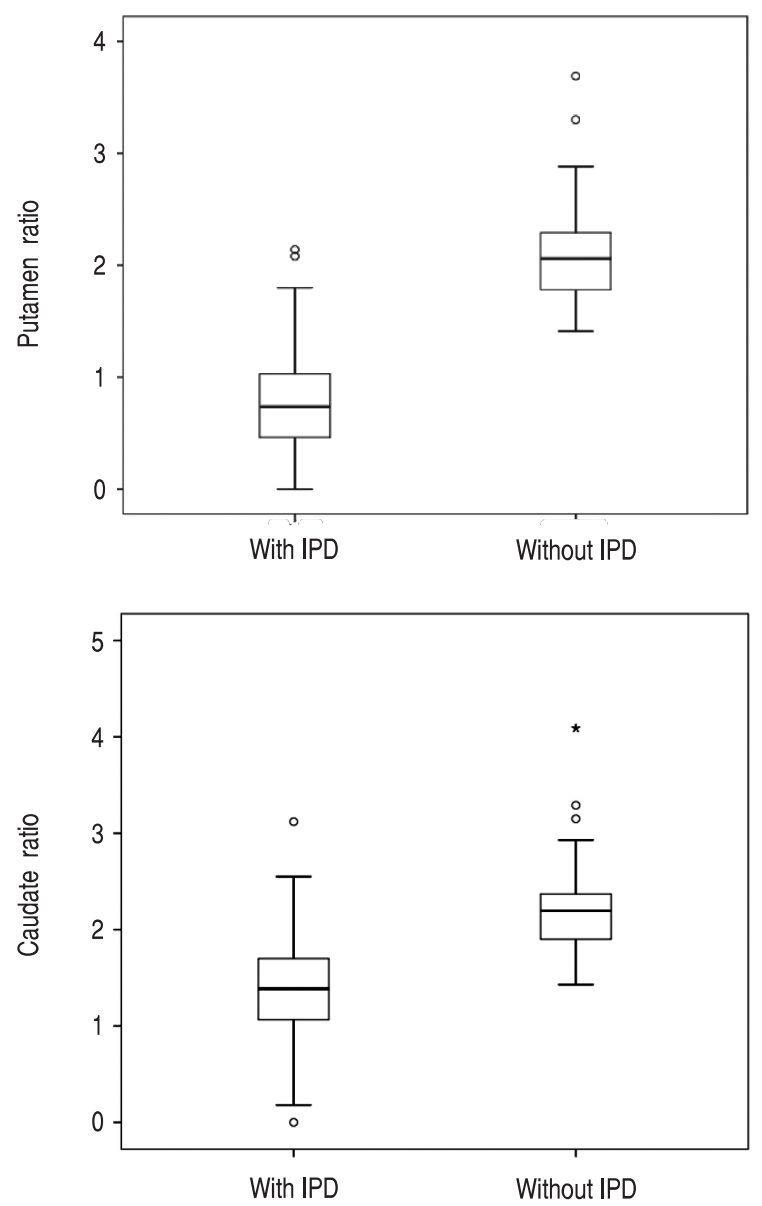

Figure 4. The uptake ratios for the putamina (A) and the caudate nuclei (B) grouped into abnormal and normal scans as determined by clinical follow-up

Table 1. Percentage of abnormal scans identified by $\mathrm{CUR}_{\min }$ or $\mathrm{PUR}_{\text {min }}$

Parameter Percentage of abnormal scans identified $(n=84)$

CURmin: 1.37 $61 \%$

PURmin: 1.35 $94 \%$ 
Table 2. Number of studies reported as equivocal (A) and accuracy (B) (defined in the text) of each assessor, with and without quantification

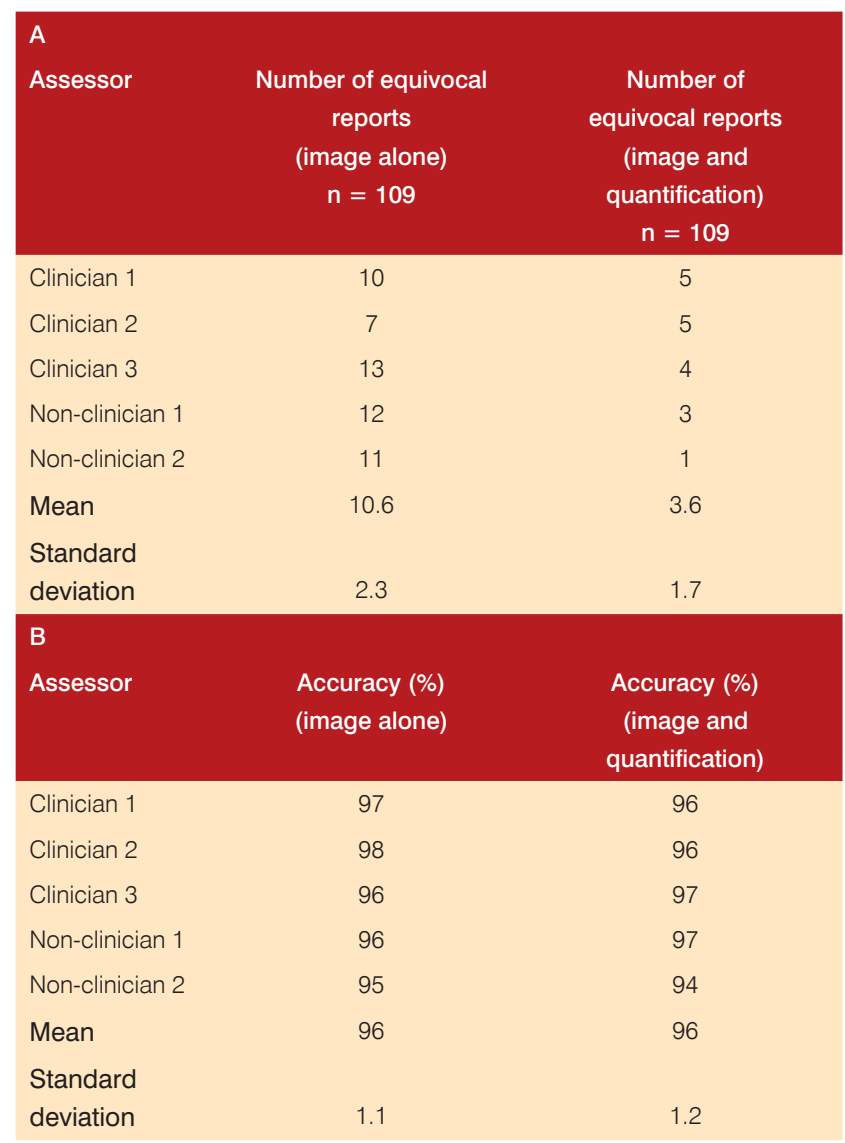

equivocal reports, with and without quantification were significantly different. $(p<0.01)$

Accuracy of reports without the use of quantification was 96\% (SD 1.1\%) and with the use of quantification was also 96\% (SD 1.2). Student's t-test (two tailed, paired) showed that there was no significant difference in reporting accuracy with and without quantification $(p=0.54)$.

Table 2A also shows that, without quantification, the highest number of equivocal reports were from the non-medical reporters. However, with quantification, this pattern was reversed.

\section{Discussion}

Our study demonstrates that the use of automated quantification of DaTSCAN studies and the use of numerical limits that define normal scans reduces the number of equivocal reports without reducing reporting accuracy. This is beneficial as equivocal DaTSCAN studies account for approximately 8\% to 9\% [4, data from our department] of cases and the most likely consequences: delayed diagnosis and rescan have important repercussions on patient outcomes.

To our knowledge, our study is the first to investigate the added value of semi-quantification in decreasing the number of equivocal results. Other published studies on semi-quantification have focused on its use in intra and inter observer variability [4, 7] and its association with visual assessment and clinical parameters [8].

Our study also has implications for the use of non-clinical reporters of DaTSCAN studies. The non-clinical reporters (physicists) had similar reporting accuracy as the clinicians however the reduction in the number of equivocal reports with the use of quantification was greatest for the non-clinicians. This suggests that non-medical staff can be appropriately trained to accurately report DaTSCAN studies and that quantification would be useful for this group.

We identified three important limitations to this study. The first is that the parameters we used $\left(C \cup R_{\text {min }}\right.$ and $\left.P U R_{\text {min }}\right)$ were defined and then tested on the same population of scans. Although this does not affect the result that the use of quantification reduces the number of scans that reporters report as equivocal, it did mean that we could not test reporting accuracy as an independent variable. To address this, we intend to retest the parameters on a new population of scans which were not originally used to define the parameters.

A second limitation is that the gold standard we used for diagnosis (clinical follow up) may have been influenced by the original report of the DaTSCAN. However the mean follow-up in our patient population was 39 months and the shortest follow-up was 18 months. We would expect an incorrect diagnosis to become increasingly apparent and questioned during such a long follow-up.

A final limitation is that striatal binding ratios decline with age [12] and so caution would have to be used when using CUR min $_{\text {m }}$ and $P \cup R_{\text {min }}$ to define normal scans in the very elderly. A more accurate approach would be to define different $\mathrm{CUR}_{\min }$ and $\mathrm{PUR}_{\min }$ for different age groups.

\section{Conclusion}

Automated quantification of tracer uptake in the striata and use of PUR ${ }_{\text {min }}$ reduces the number of scans reported as equivocal without affecting reporting accuracy.

\section{References}

1. Berardelli A, Wenning GK, Antonini A et al. EFNS/MDS-ES/ENS [corrected] recommendations for the diagnosis of Parkinson's disease. Eur J Neurol 2013; 20: 16-34

2. Felicio AC, Shih MC, Godeiro-Junior C, Andrade LA, Bressan RA, Ferraz HB. Molecular imaging studies in Parkinson disease: reducing diagnostic uncertainty. Neurologist 2009; 15: 6-16.

3. de la Fuente-Fernandez R. Role of DaTSCAN and clinical diagnosis in Parkinson disease. Neurology 2012; 78: 696-701.

4. Tondeur MC, Hambye AS, Dethy S, Ham HR. Interobserver reproducibility of the interpretation of I-123 FP-CIT single-photon emission computed tomography. Nucl Med Commun 2010; 31: 717-725.

5. Morton RJ, Guy MJ, Clauss R, Hinton PJ, Marshall CA, Clarke EA. Comparison of different methods of DatSCAN quantification. Nucl Med Commun 2005; 26: 1139-1146.

6. Badiavas K, Molyvda E, lakovou I, Tsolaki M, Psarrakos K, Karatzas N. SPECT imaging evaluation in movement disorders: far beyond visual assessment. Eur J Nucl Med Mol Imaging 2011; 38: 764-773.

7. Söderlund TA, Dickson JC, Prvulovich $E$ et al. Value of semiquantitative analysis for clinical reporting of ${ }^{123} \mid-2-\beta$-carbomethoxy-3 $\beta$-(4-iodophenyl)- 
-N-(3-fluoropropyl)nortropane SPECT studies. J Nucl Med 2013; 54: 714-722.

8. Papathanasiou N, Rondogianni P, Chroni P et al. Interobserver variability, and visual and quantitative parameters of (123)I-FP-CIT SPECT (DaTSCAN) studies. Ann Nucl Med 2012; 26: 234-240.

9. Koch W, Radau PE, Hamann C, Tatsch K. Clinical testing of an optimized software solution for an automated, observer-independent evaluation of dopamine transporter SPECT studies. J Nucl Med 2005; 46: 1109$-1118$
10. Morton RJ, Guy MJ, Clauss R, Hinton PJ, Marshall CA, Clarke EA. Comparison of different methods of DatSCAN quantification. Nucl Med Commun 2005; 26: 1139-1146.

11. Booij J, Tissingh G, Winogrodzka A et al. Practical benefit of [123l]FP-CIT SPET in the demonstration of the dopaminergic deficit in Parkinson's disease. Eur J Nucl Med 1997; 24: 68-71.

12. Nobili F, Naseri M, De Carli $F$ et al. Automatic semi-quantification of $\left[{ }^{123} \mid\right]$ FP-CIT SPECT scans in healthy volunteers using BasGan version 2: results from the ENC-DAT database. Ann Nucl Med 2012; 26: 234-240. 